\title{
Conflictos en la frontera, los derechos y las políticas de un pacto social
}

\author{
Neida Albornoz-Arias* \\ Aloisio Ruscheinsky** \\ Rina Mazuera-Arias*** \\ \& Fernando Ortiz****
}

Resumen: Los conflictos de las fronteras entre naciones y los pactos sociales establecen interacciones entre agentes sociales, derechos y el Estado democrático. El objetivo del artículo es analizar desde el enfoque sociológico, la relación entre la percepción de existencia de pactos sociales y los múltiples conflictos. Investigamos la frontera Norte de Santander (Colombia) y Táchira (Venezuela). El recurso teórico se alía con el punto de vista operativo de la investigación sobre prácticas sociales, con observación de campo y aplicaciones de encuestas. Entre los hallazgos destacamos las múltiples interacciones entre Estado y los ciudadanos, pero sin la existencia de un canal de resolución de cuestiones fundamentales en el espacio territorial y por lo tanto persisten las inconsistentes posibilidades de un pacto social ante los conflictos en el referido contexto fronterizo.

Palabras clave: Derechos. Conflictos. Pactos sociales. Contrabando. Frontera.

\section{Conflicts in the border, the rights and policies of a social pact}

Abstract: The conflicts of the borders between nations and the social pacts establish interactions between social agents, rights and the democratic State. The objective of the article is to analyze, from the sociological point of view, the relationship between the perception of the existence of social pacts and the multiple conflicts. We investigated the northern border of Santander (Colombia) and Táchira (Venezuela). The theoretical resource is combined with the operational point of view of research on social practices, with field observation and survey applications. Among the findings we highlight the multiple interactions between the State and the citizens, but without the existence of a channel for resolving fundamental issues in the territorial space and therefore the inconsistent possibilities of a social pact with respect to conflicts in the aforementioned border context persist.

Keywords: Rights. Conflicts. Social pacts. Smuggling. Border.

\author{
* Neida Albornoz- \\ Arias es doctora en \\ ciencias sociales y \\ jurídicas (Universidad \\ de Córdoba), España; \\ adscrita a la Facultad \\ de Administración \\ y Negocios de \\ la Universidad \\ Simón Bolívar, \\ Cúcuta, Colombia; \\ investigadora de \\ la Universidad \\ Católica del Táchira, \\ Venezuela. \\ Orcid: 0000- \\ 0001-7851-5985. \\ <n.albornoz@ \\ unisimonbolivar.edu. \\ co>.
** Aloisio
Ruscheinsky é doutor em sociologia, com pós-doutorado na Universitat Autònoma de Barcelona (UAB), Espanha. Professor titular do Programa de Pós-Graduação em Ciências Sociais da Universidade do Vale do Rio dos Sinos (Unisinos), São do Sul, Brasil. Orcid: 0000-0003-1297- 0795. <aloisior@unisinos. br>.
*** Rina Mazuera- Arias es doctora \\ Leopoldo, Rio Grande \\ en derecho por
}




\section{Introducción}

la Universidad de Zaragoza (España); adscrita a la Facultad de Ciencias Jurídicas y Sociales de la Universidad Simón Bolívar, Cúcuta, Colombia; docente e investigadora de la Universidad Católica del Táchira, Venezuela. Orcid: 00000002-9888-5833. <r.mazuera@ unisimonbolivar.edu. co>.

\section{**** Fernando}

Ortiz is doctor in counseling psychology in Washington State University, Pullman, Washington, United States of America; currently working in Gonzaga University, Counseling Services, Spokane, Washington, United States of America. Orcid: 00000003-3445-6332. <ortiz2@gonzaga. edu>.

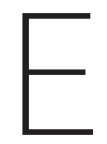
I objeto de estudio para el presente artículo son los conflictos de las fronteras entre las naciones como Venezuela y Colombia y que se establecen como interacciones entre agentes sociales, derechos y el Estado democrático. En realidad, el campo de estudio es mucho más que el punto de encuentro de dos Estados nacionales, sino también una zona de flujos transfronterizos que opera como mecanismo de abastecimiento y como aporte internacional de integración. El presente artículo tiene como objetivo comprender desde el enfoque sociológico la relación entre la percepción de existencia de pactos sociales y la vida cotidiana de búsqueda de derechos según algunas variables, como entre otras la ocupación, nivel educativo y edad, la contribución en el contrabando y confianza en instituciones. De acuerdo con este objetivo, se permite a los autores observar, investigar, describir y explicar algunas cualidades de los residentes de las ciudades nombradas con relación a sus experiencias de vida en un espacio fronterizo. Para visualizar estas vivencias bajo la óptica de los actores sociales se aborda desde los enfoques que se construyen a partir de sus visiones de mundo o de las representaciones sociales sobre las actividades transfronterizas, legales e ilegales, de los sujetos ubicados en una zona de conflicto.

Se inicia de la visión del habitar, del trabajar, del traficar como indicadores que ofrecen o sustentan un abordaje de la complejidad de los fenómenos sociales, de cómo se construye socialmente una realidad que se instaura en un determinado territorio. Donde los condicionantes políticos, geográficos, sociales y culturales que se agregan al territorio, lo hacen en forma permanente o temporal, generan distintos resultados en la negociación de los conflictos.

El problema de la investigación subrayaconocer la realidad de los conflictos en el cotidiano de los agentes sociales fronterizos y la demanda por derechos multidimensionales. En la estrategia metodológica se incluye un matiz sociológico en la cual se considera a los actores locales como parte fundamental de construcción de la configuración de la ciudad. En este texto, se utiliza la doble perspectiva de auscultar las estrategias de actores sociales y delas contingencias políticas do contexto, bajo el efecto de las posibilidades de combinación e intercambio de investigación sobre las fronteras múltiples. Bajo la primera,se analiza percepciones, perspectivas y diferencias en la visión del mundo, inclusive información circulante en las redes sociales. Se usan tales recursos de análisis para comprender la orientación de la acción político-social en el contexto del contrabando y de la fragilidad de un contrato social. Por el trabajo con la población y con una observación esmerada del cotidia- 
no, se puede enfatizar que son numerosas familias las que sostienen su economía con estos ingresos, siendo de interés para los investigadores observar la percepción de los encuestados ${ }^{1}$.Los componentes que conforman la realidad en conflicto son relaciones sociales desiguales y a nosotros interesa destacar una visión acerca de las percepciones sobre lo legal y lo ilegal en la búsqueda de derechos de ciudadanía. La idea es captar relaciones por medio de un lenguaje implícito en los actos de múltiples violencias practicadas y a partir de este sesgo se genera la reflexión: "hablamos de investigar un actor actuando, analizar una perfomance en plena escenificación" (Sandoval, 2013: 42). Para la perspectiva de la acción

cuando investigamos el sentido de una práctica social, este no sólo radica en las narraciones que elaboran los individuos, sino que también proviene de los contextos y los objetos materiales que tienen incidencia en el desarrollo de dicha práctica social (Sandoval, 2013: 43).

En otros términos, indagar la red de sentido de la cual los sujetos son parte activa, el significado atribuido a un contrato social.

Bajo otro aspecto, en una perspectiva complementaria,un estudio de carácter exploratorio se utilizó una encuesta dirigida a personas entre 18 y 69 años tanto en territorio colombiano como venezolano. Se empleó un muestreo representativo ${ }^{2}$ de la población: 2.394 personas en Norte de Santander y 1.398 personas en el Táchira. Además, en el mapeo fronterizo se desea que la población declare sus propias experiencias por medio de un cuestionario, para esto se incluyeron indicadores como datos demográficos, vivienda, movilidad interurbana, posibles comprensiones de las funciones públicas de acuerdos, del fenómeno de control por el Estado nacional, entre otros temas. Pero, no todas estas temáticas de una larga investigación caben en un artículo. Se tiene visualizada la existencia de visiones múltiples o diferencias significativas entre los segmentos sociales e individuos. La construcción del texto también se sirvió de las contribuciones y aspectos contemplados en la investigación sobre la institucionalidad en Táchira (Venezuela)(García et alii, 2016)en relación con las prácticas del contrabando examinando los siguientes elementos ${ }^{3}$ : Estado de Derecho; capital y cohesión social; conciencia y cultura cívica ciudadana. Los investigadores en extenso trabajo hicieron un levantamiento de información en todos los municipios del estado Táchira. En el presente texto se enfoca de modo muy privilegiada la opinión sobre la percepción de existencia de pactos informales o acuerdos entre personas y/o instituciones en la zona de frontera.
1. Los datos analizados en el texto provienen de investigaciones realizadas en territorios fronterizos

i. Norte de Santander (Colombia) por la Universidad Simón Bolívar, sede Cúcuta; $y$

ii. Estado Táchira (Venezuela) por el Observatorio Social de la Universidad Católica del Táchira, sede San Cristóbal.

2. Dicha información fue recogida en los cuarenta municipios del Departamento Norte de Santander y los veintinueve municipios del Estado Táchira, entre los meses abril a junio de 2015 .

3. La colectividad puede conocer los resultados en su versión en línea: $<$ www.ucat.edu.ve/ oset>. 
4. En sus palabras: "Una organización gobernante será considerada política en tanto y en cuanto su existencia y orden sobre un territorio esté continuamente salvaguardada por la amenaza y la aplicación de la fuerza física por parte de su personal administrativo, Una organización política obligatoria con operaciones continuas será llamada estado en tanto y en cuanto su aparato administrativo mantenga para sí, con éxito, el monopolio del uso legítimo de la fuerza en la aplicación del orden por él establecido" (Weber, 1978: 54)

\section{El repertorio de fronteras, territorio y políticas públicas}

Las aportaciones teóricas y metodológicas en una perspectiva histórica sirven para comprender al proceso de socialización en la frontera con el sentido político-social de construcción de la realidad. El territorio en la frontera es simbólicamente ocupado por los actores sociales (individuales y colectivos), considerado también como un área vital geopolíticamente. Es también una territorialidad delimitada donde se fomentan relaciones de poder y gestión del Estado, de personas y organizaciones. La dinámica colectiva, que se instaura en los conflictos fronterizos, depende de referencias y motivaciones con sus relaciones de tensión y de reciprocidad (Gaiger, 2014). En esta forma, se atribuyen valor a las prácticas sociales que no imponen a los migrantes un yugo directo de relaciones de subordinación. En el territorio se desprende afectividad e identidad, negociación y lealtad por parte de los actores locales, los cuales tienen intereses, percepciones, valores, conflictos y demandas distintas. La formulación de Weber acerca del criterio para reconocer una organización política como un Estado ${ }^{4}$ requiere presencia.

En el espacio territorial cohabita con la noción de ciudadanía. Es donde existen relaciones de intereses, con tipos de circulación de capitales, desde el financiero a lo simbólico, es decir, un espacio público. En el espacio social existen relaciones de poder, ejercicio de la fuerza, formas de resistencia, movimientos múltiples, solidaridad y utopías. En el espacio se tejen imágenes asociadas con relaciones grupales, con distintas representaciones que dependen de las circunstancias de los individuos y de los colectivos (Taylor, 2007). El mismo, se construye con base en experiencias, acciones, discursos, dimensiones simbólicas de un lado y de otro los condicionamientos y contradicciones que, al ser analizados, permiten la diferenciación de lugares y así pueden identificarse y atribuir funciones, apropiarse, marcar "límites, linderos, fronteras y demarcaciones" (Brenna, 2012: 84).

En fin, en las modificaciones históricas lo relevante es el enlace e interacción de los integrantes del espacio público. La noción de espacio público posee algunas dimensiones, tales como, los aportes políticos, la negociación entre actores sociales, los valores culturales y aun conforma una esfera cotidiana del sujeto. En la presente mirada, territorio viene tanto cargado de elementos objetivos y palpables, como traspasado por elementos subjetivos y palpitantes. Al mismo tiempo se plantea en pauta discusiones de carácter político y cultural, social y económico.

El concepto frontera tiene muchos significados, uno de ellos se refiere a la región que separa a dos o más países y comprende el límite y borde. Una frontera tiene dis- 
tintas concepciones que pueden relacionarse con los conceptos espacio y territorio. Todavía hay que considerar el hecho que las fronteras territoriales de delimitación de los Estados nación (borders), aunque no necesariamente coincidentes, coexisten con fronteras socioculturales(boundaries). Estas últimas, como ocurre en el contexto en estudio, a veces se superponen y se prolongan más allá de las primeras fronteras. La movilidad y la diferencia, hostilidad y acogida son asuntos íntimamente relacionados como el movimiento de personas, especialmente el movimiento de otros como extraños, a menudo evocó la erección de fronteras en el doble sentido señalado arriba (Cunningham \& Heyman, 2004). En este sentido, la libertad y las restricciones se hacen, deshacen y rehacen.

En los procesos transfronterizos se argumenta que los parámetros de fronteras geográficas, políticas y culturales se entrecruzan, particularmente ilustran contradicciones, paradojas, conflictos de poder, debilidades del estado-nación en la contemporaneidad (Álvarez, 1995). Como resultado de la observación de campo en el doble sentido de la frontera se destacan diversos resultados, especialmente como procesos que delimitan, fiscalizan y restringen el movimiento de personas y la circulación de bienes de consumo. La construcción de las fronteras nacionales suena como el establecimiento y las delimitaciones del mercado de circulación de mercancías.

Para la presente investigación es posible considerar y entender también la noción de frontera situada entre lo legal y extralegal, entre los sujetos de la acción que transcurre en el cotidiano y las instituciones que representan al Estado. Con el fin de presentar la dimensión operativa y los fines contingentes, Matteucci (1988) distingue "Estado-árbitro", "Estado protector" o "Estado productivo" que provee los bienes públicos al mismo tiempo que dispone de poderes discrecionales. Por una parte, en la perspectiva dinámica, más que el límite o línea divisoria es un transbordo a la zona contigua o el encuentro con el otro.

La segunda dimensión del concepto de frontera tampoco representa una "línea", sino que la frontera se refiere a un área de integración/separación gradual e incluso, a veces, simultánea. Esto quiere decir que la frontera es una zona de transición entre territorios. Es precisamente en la frontera donde se encuentran e interactúan, y hasta se funden, las diferentes formas y normas que caracterizan cada territorio (Sánchez, 2015: 178).

La frontera va más allá del límite, es un espacio de integración y/o separación, una zona de transición entre los territorios, donde interactúan normas y pactos que identifican cada territorio, donde hay además una membrana penetrable (Taylor, 2007; Sánchez, 2015). La frontera también demarca las restricciones à libertad o la 
expansión de la economía capitalista, al mismo tiempo significa el espacio propio de encuentro de sociedades y culturas diferenciadas. Por esta ocasión de epopeyas diversas, de acuerdos y pactos, como el lugar de la búsqueda desenfrenada por una ventana de oportunidades, pero también del genocidio, extorsión o de la masacre de los humildes. Desde este punto de vista, para Martins

frontera esencialmente el lugar de la alteridad. Esto es lo que hace de ella una realidad singular. Un lugar del descubrimiento del otro y del desencuentro (Martins, 1997: 147).

Por una visión de la dinámica de las relaciones sociales,

\begin{abstract}
la dialéctica que se desarrolla en una definición concreta entre los conceptos... nos permite asumir algunas cualidades que los unifican para relacionarlos con los usos y prácticas que adoptan los individuos dentro de una ciudad determinada, la cual permite vincular las formas que suceden en la relación del espacio urbano y social con el territorio a través de las prácticas que se generan en una ciudad específica (Murrieta, 2017: 133).
\end{abstract}

Hay aspectos singulares en las fronteras en América Latina y El Caribe, en el caso de la frontera de Colombia y Venezuela, ha existido búsqueda a grupos armados y en acción al margen de la ley. Ante este fenómeno han robustecido la presencia de fuerzas armadas, pero según Perus (2005), debido a la porosidad de misma, surgen y persisten territorios dispuestos para la producción y tráfico de drogas y contrabando.

El tercer concepto de políticas públicas se refiere a los objetivos de bienestar a alcanzar a través dela acción del Estado. Cuando desde la óptica institucional se pretende abordar las relaciones en el espacio, como en el caso presente, valorar la acción en el campo de las políticas sociales es reconocer la dinámica de las territorialidades Colombia/Venezuela (Sánchez, 2015). Es posible argumentar, que las políticas potencialmente resultan de los procesos y capacidades institucionales y se convierten en mecanismos políticos o administrativos con influencias antinómicas en muchos aspectos del cotidiano de los ciudadanos, en la amplitud de aspectos políticos, económicos, sociales y culturales.

Para justificar el examen de las políticas públicas como frontera es posible afirmar que al inicio del Siglo XXI, grandes fracciones de la población en América Latina, son pobres, excluidos e informales; los mecanismos de la informalidad presente queda unida a la exclusión del empleo constante, ingreso habitual y acceso a instituciones sociales del Estado democrático de derecho. En las entrañas de los órganos estata- 
les, la aporía del control de las actividades del contrabando ha empezado a ser foco de atención para el diseño de políticas públicas regionales. Además, el fenómeno en el nuevo escenario internacional ha sido estudiado por las ciencias sociales y hoy un tópico de investigación reconocido. Sin duda, constituye un actualizado problema social fronterizo a ser abordado en sus diferentes costados.

Paradójicamente, la frontera como pasaje y como control es espacio de excepcionalidad donde se expone como visible la creatividad de los marginalizados.

\begin{abstract}
es aquí donde formas alternativas de acción económica y política son instituidas. Sugerir que los márgenes son espacios de creatividad no es decir que las formas que adquieren la política y la economía en estos, las cuales generalmente son formadas por la necesidad de sobrevivir, no estén cargadas de terribles peligros (Das \& Poole, 2008: 34).
\end{abstract}

Por la lógica de la racionalidad nadie se dedica a una actividad peligrosa para su propia vida si tiene un trabajo que le garantiza bienestar. En este panorama, el énfasis puesto en las adversidades frente al bienestar por la parte de contrabando tiende a eclipsar las peculiaridades regionales de labor de los ciudadanos por la sobrevivencia (Flores \& Pacheco, 2017). Las brechas de creatividad se han construido históricamente y que se asocian a las cuestiones expuestas, suelenser invisibles en los análisis y en los diseñadores de políticas públicas. Nos referimos al diseño de políticas inclusivas y con enfoque de seguridad de los derechos.

\title{
Los conflictos, la acción del Estado y contrabando en la frontera
}

Cada espacio fronterizo posee características que permiten construir una singularidad de ellos y al mismo tiempo trazos equivalentes a otras experiencias. De este punto de vista, cada escenario fronterizo es peculiar, como únicas son las fronteras que lo conforman, pero entre Colombia y Venezuela no es la excepción por la presencia de estratégicos actores y la búsqueda de bienestar social delante políticas desarticuladas y asimetrías socio-culturales persistentes. Allí se cruzan "universos simbólicos diferentes y desiguales", que hacen vida en ella y participan en una "economía de frontera" variada y dividida entre bienes materiales y inmateriales, entre "mercados legales e ilegales" y en el cual intervienen "actores transfronterizos" (Carrión \& Espin, 2011: 13). Entre otras dificultades, en este territorio existe el contrabando, de causa multidimensional, como por las distorsiones del mercado que provienen de diferencias económicas y jurídicas. La multidimensionalidad se reporta aún a las diferencias de precios de productos y de acceso al consumo entre 
FIGURA 1

MAPA DE LA FRONTERA COLOMBO-VENEZOLANA



Fuente: Naciones Unidas, Colombia. Disponible en: <http://nacionesunidas.org.co/log/2015/09/24/formede-situacion-no-8-colombia-situacion-humanitaria-en-frontera-colombo-venezolana/>.

las regiones; el contrabando siempre es relativo a las formas de control social, al mismo tiempo que se encuentra saturado de simbologías de la publicidad y de las narrativas populares; no por último, la complicidad entre ciudadanos en circulación y agentes gubernamentales.

5. Otras

informaciones y datos sobre el territorio fronterizo en Caraballo (2017).
La frontera entre Colombia y Venezuela (Figura 1) comprende una extensión de $2.219 \mathrm{~km}$, pero el análisis se centra en el Departamento Norte de Santander (Colombia) y Estado Táchira (Venezuela), donde existe el principal espacio habitable o concentración poblacional urbana, transbordo de mercancías y densidad institucional de todo el cordón fronterizo ${ }^{5}$. La ubicación de la extensa frontera ofrece la posibilidad de esculpir numerosas rutas de contrabando, no sólo entre estas dos ciudades, sino por extensos territorios prácticamente baldíos.

Según datos del Departamento Administrativo Nacional de Estadística (Dane, 2015) informan que en la proyección de 2014 Cúcuta tiene una población de 650.011, de los cuales $25 \%$ son rurales y el Departamento Norte de Santander tiene una población de 1.355.787 de habitantes. Del centro de la ciudad a la frontera son cerca de 12 km. Táchira tiene 1.168.908 habitantes (INE, 2011) y el municipio San Cristóbal una población de 646.721 y se localiza cerca de $50 \mathrm{~km}$ de la frontera. 
Las autoridades a menudo postulan operaciones contra el contrabando y la aprehensión de contrabandistas como objetivos clave de esfuerzos de interdicción, así como la justificación para intensificar las actividades de fiscalización para sosegar sectores de actividad comercial legalmente establecidos. Es importante, pues, interrogar cómo funciona el contrabando en grandes cantidades. Este es típicamente orquestado por emprendedores organizados, debido al grado de planificación, costo, equipamiento necesario, conocimiento de rutas y de agentes de control social.

Por otra parte, hay factores de orden nacional que afectan a la región, presentados por Bustamante (2011) y que no son objeto de consideración; igualmente, a los miles de colombianos que son considerados como desplazados viviendo en territorio venezolano. Sin embargo, la autora pondera que

las diferencias en las políticas macroeconómicas también han tenido impacto negativo para los habitantes de la frontera. El control de cambios en Venezuela, la fijación de un valor único del precio del dólar, la creación de la Comisión Administradora de Divisas (Cadivi), así como las medidas para controlar el contrabando de extracción de alimentos, y el mantenimiento de los combustibles (gasolina y gasoil) a precios artificialmente bajos han llevado a la pérdida del poder de compra del bolívar respecto a la moneda extranjera. Ha permitido la creación de mecanismos engañosos en la frontera dirigidos a la obtención de la moneda extranjera con los dólares autorizados por Cadivi, al contrabando de alimentos y combustibles al punto que se produce desabastecimiento de los mismos en el lado venezolano de la frontera (Bustamante, 2011: 214).

La situación y problemática descrita anteriormente refleja la falta de acuerdos entre los gobiernos para diseñar estrategias comunes y cooperar para aclarar la articulación económica, política y de inseguridad, entre otras. El tráfico de narcóticos y el contrabando de combustible, alimentos, medicinas, entre otros, son los negocios ilegales que identifican esta frontera (Carreño, 2014; Riaño-Garzón et alii, 2018). La mayor fuente de ingreso del Norte de Santander es el comercio lícito e ilícito con Venezuela, especialmente con las ciudades venezolanas San Antonio, Ureña que bordean la frontera y San Cristóbal que es la capital del Estado Táchira. Sobre las relaciones socio-espaciales en esta región, Carreño (2014) y Caraballo (2017)señalan que siempre ha existido el paso de colombianos y venezolanos de un lado al otro, como un fenómeno cultural y de la economía.

Para Stein et alii (2015: 14), el contrabando es un delito aduanero por excelencia, en el cual no sólo concurren conductas evasoras de impuestos, sino se "evidencia una 
6. Sólo a modo de ilustración de la extensión del fenómeno: la tenencia de tierras urbanas y rurales, acceso al agua, lavado de dinero, tráfico de drogas, de personas, armas,alimentos, combustibles, medicinas,órganos, bebidas alcohólicas, contrabando de vida silvestre, tecnologías entre otros aspectos. red de corrupción que contamina de manera no focalizada sino global a todos los estamentos involucrados y a muchos de sus actores".

Las dinámicas sociales, culturales y económicas de la frontera están inmersas entre lo legal e ilegal. Dentro de lo ilegal se cuentan los delitos ${ }^{6}$, identificando un mayor número de conflictos transnacionales. De acuerdo con Ávila et alii (2012), esta dinámica comprende el contrabando y la presencia de grupos armados que de manera organizada y con el uso de la violencia, soborno, extorsión obtienen beneficios económicos y políticos.

En la encuesta se preguntó a las personas ¿qué productos considera usted son los de mayor objeto de contrabando (seleccione 3)? Las respuestas provienen de colombianos y se refieren a productos que salen de Venezuela. Son estimados los principales productos de contrabando: Productos de primera necesidad (Canasta familiar) con 2127 respuestas o 88,8\%; Gasolina y otros combustibles con 1648 respuestas o 68,8\%; productos agropecuarios con 1218 respuestas o 50,8\%; productos para limpieza del hogar con 959 o 40,1\% y muy abajo vinos y licores con 466 respuestas o 19,5\%. En la dinámica socio-espacial, hay que ponderar que se encuentra en vigor un límite ofuscado entre lo legal y lo ilegal en las prácticas cotidianas. Acá acompañamos el significado de los términos de acuerdo con Carrión y tenemos la convicción de su adecuación.

\begin{abstract}
este proceso produce una mutación importante en términos de los actores locales; se pasa del contrabandista, que en esencia es un especulador que traslada las mercaderías de una economía hacia otra aprovechando las ventajas comparativas que tiene una sobre la otra y, generalmente, lo hace obviando el pago de aranceles; al traficante, que es la figura social de un delincuente, que traslada hacia el otro lado de la frontera sujetos y objetos ilegales (personas, armas, órganos, droga) que están inscritos en circuitos de intercambio internacional (Carrión, 2011: 103).
\end{abstract}

En los negocios pueden distinguirse varias modalidades:

i. tránsito de mercancías diversas con pago de impuestos de acuerdo con la legislación vigente;

ii. el paso de pequeñas cantidades (menos de 12 kilos, unidades o litros), de alimentos y productos de consumo masivo se debe al diferencial cambiario entre las monedas de Colombia y Venezuela;

iii. el de grandes cantidades de mercancías sin pagar aranceles están relacionadas a organizaciones criminales que tienen el monopolio y 
lucro, y combaten por el control de rutas informales para el tránsito de mercancías (Albornoz et alii, 2016).

iv. grupos irregulares que actúan, por veces, en complot con funcionarios estatales o cuerpos de seguridad que refleja un comportamiento de cooperación, entre funcionarios públicos y contrabandistas en la frontera.

Sin lugar a dudas, para encetar las prácticas distintas del contrabando en la frontera, hay que conferir la existencia de una asignación de status público a grupos de interés (Offe, 1981). Las prácticas de contrabando son promovidas por diversos actores, a pesar de las barreras existentes (institucionales y políticas) a la entrada o intercambio de mercancías como es señalado arriba, es difuso en las dinámicas socio-espaciales. Esta dinámica, que tiene una fuerte implicación de un componente político de la acción de los Estados nacionales, se corresponde con un problema alentado por la diferencia de valores y distintas mercancías en la circulación. Las asimetrías están manifiestas en el diferencial cambiario, costo de bienes y servicios, con controles de cambio y de precios, política de subsidios y regulación, entre otras. Esclarece Sánchez:

Estas dinámicas inciden, con particulares grados de relevancia, a diferentes escalas de nuestra existencia social. Sin embargo, cuando desde las instituciones estatales se realizan estas prácticas de construcción de territorios, y, por tanto, involucrando identificación de límites y bordes, el entendimiento del territorio y de las dinámicas de territorialidad es vital. En este sentido, la interpretación territorial y los bordes y límites que de ésta emanen implican particulares concepciones y formas de organización y ordenamiento del espacio, lo que de forma directa incide en relaciones y reacciones sociales (Sánchez, 2015: 177).

No es de hoy la situación que continúa potenciando la práctica del contrabando a pesar de los esfuerzos de las autoridades por controlarlo. Paradoxalmente, en este espacio fronterizo hay la fluencia de relaciones sociales sobre las cuales inciden las políticas públicas, como acciones de gobernanza y todas las consecuencias de este fenómeno histórico (Navarro \& Pérez, 2012). En este sentido, la discusión agrega una realidad dispar pautada en medio del entrelazamiento de conflictos, políticas públicas y gobernabilidad. Con esto se plantea un cuestionamiento y una hipótesis relevante en el abordaje: los mecanismos de la gobernanza no dan cuenta de la efectuación de las políticas públicas, ordenando el mercado laboral con el enfoque de derechos, en una territorialidad impregnada por conflictos y contrabando. 
7. De acuerdo con Carrión (2011) los diversos niveles de violencia hacen con que venía a aparecer nuevos delitos y lo que, a su vez, produjo la emergencia de nuevos actores vinculados, por ejemplo, el paso del contrabandista a narcotraficante $y$ el aparecimiento de otros como mulas, sicarios y otros más.
Los actores sociales son de alguna forma determinantes en la formulación de una perspectiva política que sostiene un pacto social. En otros términos, la atribución de prestigio social y público a los grupos de interés por parte del Estado nacional para establecer una legitimidad de las negociaciones de los conflictos en cuanto a los accesos a los medios de sobrevivencia de los ciudadanos. Un pacto social emerge como un proceso de negociación entre partes que sí reconocen como agentes activos de un proyecto. En este sentido, nosotros tenemos una interrogación: ¿̇los intereses de bienestar pueden ser uno de los objetos de la negociación entre los agrupamientos sociales y las instancias del poder público en la zona fronteriza? La emergencia de un pacto social, depende la capacidad de intervención política, visando a la atenuación de los conflictos (Offe, 1981), con una visión republicana y de alguna forma conducirá a la imposición de restricciones y disciplina o acatamiento a una orden colectiva.

En algunas fronteras existen relaciones entre los actores locales que demuestran la dinámica de la sociedad civil, en sus formas de organizarse para combinar acciones legales e ilegales ${ }^{7}$, vínculos económicos o amistad, en redes locales o transnacionales y que conforman la cotidianidad y la conflictividad. Los márgenes, las fronteras y las políticas públicas no son inertes, pero son cargados de dinámicas como rastreamos entre Colombia y Venezuela. El examen de las zonas fronterizas en el contexto de los estados nacionales es algo concerniente a la indicación de lo que queda dentro y de lo que queda fuera, razón por la cual son justificados los puntos y mecanismos de control y de quien tiene derecho al acceso a los bienes sociales, sean materiales e inmateriales. En un cierto sentido, los mecanismos de control, no solo en las fronteras atraviesan el cuerpo político del Estado.

\footnotetext{
Las fronteras y los puestos de control, como hemos visto, son espacios en los cuales la soberanía, en tanto derecho sobre la vida y la muerte, es experimentada de un modo potencial, creando efectos de pánico y un sentimiento de peligro (Das \& Poole, 2008: 34).
}

Además de estas afirmaciones, las políticas públicas, por su propia definición, tienen por disposición la ampliación de las fronteras de la ciudadanía.

\section{La efectividad y las reglas de juego de las relaciones sociales}

Los pactos se instituyen como acuerdos culturales, políticos o jurídicos entre individuos libres o grupos sociales, que anhelan alcanzar relaciones sociales para una convivencia. En este momento no establecemos la distinción entre pacto y contrato 
social. De acuerdo con Matteucci (1988) con un pacto social ${ }^{8}$ se anhela hacer cesar la tensión entre los intereses particulares y los generales, concordando que la justicia también tiene una utilidad mediante el establecimiento de reglas del juego. Para ello hay que llevar en consideración lo que Sánchez pregona:

El territorio es una entidad espacial que sirve como instrumento de comunicación que visibiliza y hace tangible estructuras sociales, tales como autoridad, identidad, derechos, aspiraciones, prejuicios, entre muchas otras (Sánchez, 2015: 176).
8. De acuerdo con Matteucci (1988),

se denominó al documento Declaración de los derechos humanos sólo porque la palabra contrato o pacto social parecía demasiado revolucionaria.

Así, entonces, la diversidad de dinámicas sociales involucradas se concretiza como relaciones sociales de convivencia.

Al respecto para la legitimidad de las reglas del juego, hay un acuerdo de voluntades y de responsabilidades entre distintas categorías sociales que desencadenan luchas como grupos de presión por la apropiación de los bienes producidos por la sociedad. Por lo tanto, pacto es consentimiento, del cual deriva el poder político y los derechos, condición para asegurar la cohesión social a través de la negociación y regulación de conflictos.

La instauración del poder político legítimo resulta,para una situación de Estado de derecho, de un pacto o convención entre distintas facciones que se encuentran al límite de luchas como fuerzas sociales (Bobbio, 1990). Los pactos representan la dimensión histórica y racional en relación con fines, así como una tensión en tiempo de espera(stand by time) o es el momento en que las personas privilegian la cooperación. Cobra sentido así la afirmación de Bobbio en cuanto que

la vida política se desarrolla mediante conflictos que jamás son resueltos definitivamente, cuya solución se da mediante acuerdos momentáneos, treguas, y aquellos tratados de paz y con las constituciones (Bobbio, 2008: 146).

En este sentido, hay que entender que para nombrar algo como un pacto social ante el contrabando incumbe incluir derechos fundamentales enumerados por los ciudadanos.

En la Tabla 1 se presentan los resultados de la encuesta con la visión según la ocupación u oficio: si los ciudadanos visualizan en la zona de frontera la formación de pactos sociales informales (acuerdos entre personas y/o instituciones). En realidad, si sumamos los que señalaron muy de acuerdo y de acuerdo, tendremos alrededor de $70 \%$ en todas las ramas de oficios, lo que de hecho es sorprendente en la medida 
TABLA 1

SE VISUALIZAN PACTOS SOCIALES INFORMALES

(ACUERDOS ENTRE PERSONAS Y/O INSTITUCIONES) EN LA ZONA DE FRONTERA, SEGÚN OFICIO

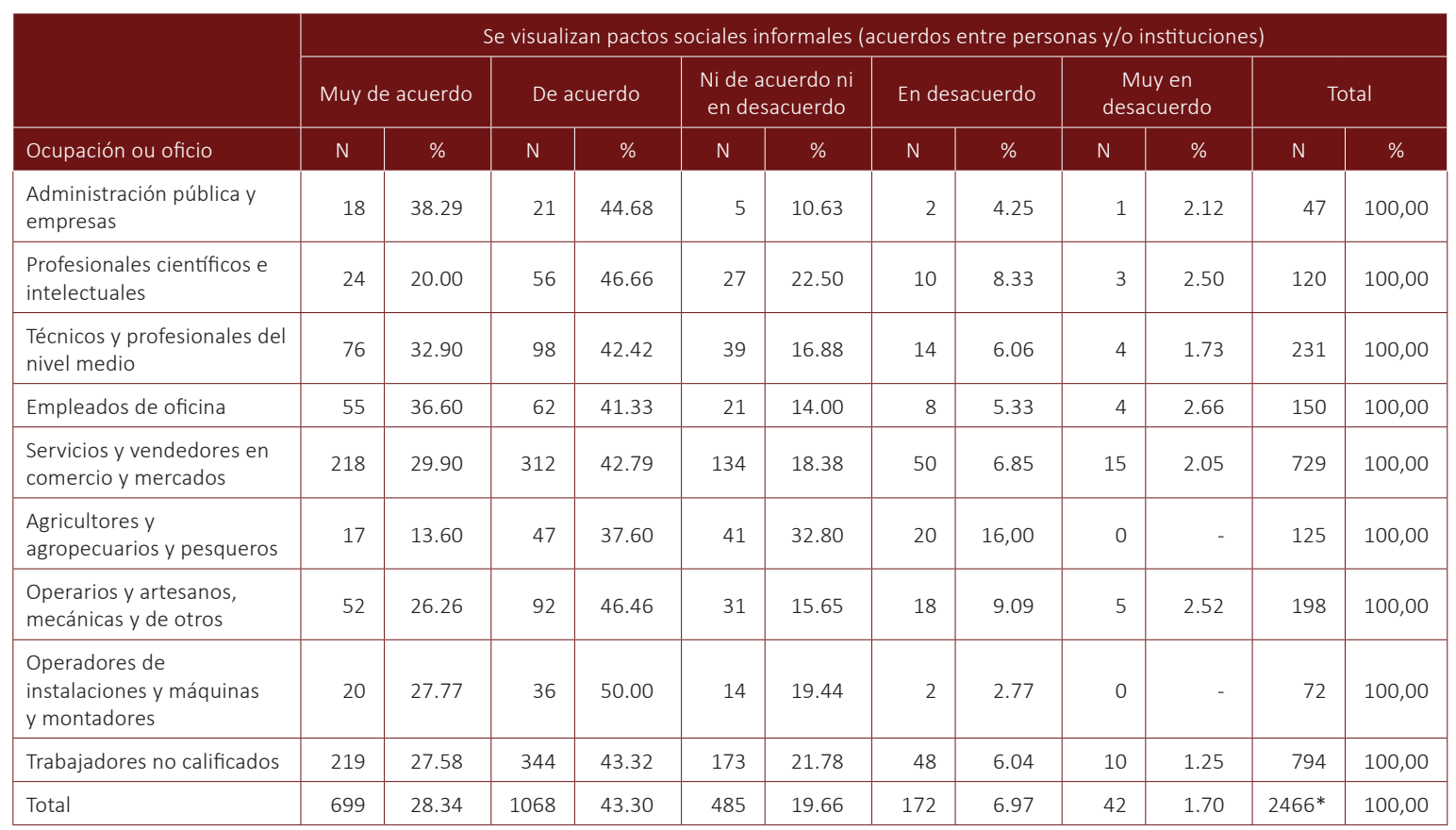

* Resultado proveniente de los encuestados que manifestaron estar ocupados al momento de ser consultados.

Fuente: elaboración propia. Datos provenientes de las encuestas aplicadas en Norte de Santander y Táchira.

9. De acuerdo con Cunningham (2012), son usuales los estudios sobre aspectos económicos, culturales, sociales y políticos de las fronteras, pero inusual es relacionar el contrabando con un complejo de relaciones con los bienes naturales, incluyendo procesos y sistemas ecológicos. en que se imagina que la aprehensión de la realidad varía de acuerdo con la profesión, los niveles económicos, el nivel de enseñanza, entre otros.

En su conjunto los actores del escenario fronterizo se involucran en compromisos y en los cuales persiguen intereses que puedan estar en conflicto. Pero, se adhieren a los mismos, ellos han encontrado el significado y la utilidad de estos instrumentos políticos (Avdagic, 2011). Tal es el desempeño de la herramienta de los gobiernos para el diseño de políticas efectivas en condiciones de especial dificultad ante el contrabando y frente a la inminencia de grandes desafíos económicos, culturales y políticos ${ }^{9}$ que requieren ajustes de largo alcance. La Tabla 1 destaca variables incorporadas a lo cotidiano, además de dimensiones que se refieren directamente a actitudes y experiencias de los ciudadanos relacionadas a las relaciones sociales fronterizas como la inseguridad, así como el objeto directo del presente trabajo en cuanto al nexo entre derechos y pactos, otras variables también están implícitas.

En relación con políticas públicas, inclusión social y conformación de pactos sociales, Sánchez (2015) destaca a su relevancia: en este sentido, una de las cosas con mayor 
significado en los procesos de territorialidad es si se está dentro o fuera de ésta. Si una persona se siente "dentro" en términos territoriales, a su vez se siente segura y no amenazada, protegida y no expuesta. En especial, observadas las contingencias de la posibilidad de que los ciudadanos visualizan la existencia de pactos (Tabla 1), en realidad, es perceptible que la opción "Ni de acuerdo ni en desacuerdo", con cerca del $20 \%$ de las respuestas, significa un distanciamiento de la temática, siendo que entre agricultores y agropecuarios y pesqueros este índice alcanza el 32,8\%. Los ciudadanos entrevistados que viven en la efervescencia de los hechos poseen una dificultad para discernir o experimentar las características peculiares o las identidades circulantes, de otro lado una dispersa autoridad estatal entre diversos agentes y agencias para insertar seguridad.

El florecimiento de la seguridad en la frontera como fuente y como objeto del pacto social son mutuamente constitutivos y ambos con presencia ambigua. En las principales travesías el desafío logístico aflictivo está en la atención efectiva al contrabando, esto, de acuerdo con Heyman (2004), pone en juego procesos sociales que tienden a ser complejos, ambiguos e implican desigualdades de derechos y tratamiento. Otro factor político que se destaca es la legitimidad y la fuerza política del gobierno local y nacional porque esto condiciona o refuerza el apoyo exógeno (Avdagic, 2008) para las políticas del control social. Dado el alcance dela noción de compromiso, de modo singular en la frontera, los pactos sociales al respecto del contrabando son la expresión de un proceso de intercambio político continuo entre gobierno, ciudadanos e interlocutores sociales o entre las interacciones entre actores sociales de la sociedad civil. Esto conforma un juego que se desarrolla en el ámbito institucional en el que cada cual controla recursos o intereses (Oberg et alii, 2011) y demarca sus posicionamientos. Dichas reglas son más o menos reconocidas o institucionalizadas y las organizaciones son los jugadores. En la frontera el gobierno intenta un control de la legislación, de los impuestos y del gasto público, todavía interrogamos ¿porqué esto se encuentra fragilizado?

En la Tabla 2 la actitud frente al fenómeno del contrabando es mayoritariamente caracterizada por la paciencia, indiferencia y resignación, por cerca de $76 \%$ dentro de los que declararan que conocen las acciones tomadas por parte del estado para minimizar dicha práctica. La interacción, así como el conflicto es parte del cambio social y los actores sociales, dados ciertos parámetros institucionales, tienden a optimizar el uso de la violencia ${ }^{10}$ obteniendo resultados diversos. Paradójicamente, la adhesión a las prácticas ilegales, como el contrabando, puede ser pensada como una forma de hacerse presente en la disputa por derechos. Para algunos autores, se trata de comprender el uso de violencia para alterar la estructura de derechos o su exclusión existente, así como si hay adhesión al uso de la influencia política. Sin embargo, para
10. Afirma Carrión, (2011: 88) con datos de 2008, "sobre Tasas de Homicidios en las fronteras Latinoamericanas, se puede observar algunas cuestiones interesantes $y$ ciertas constantes globales a las regiones fronterizas de América Latina. Por ejemplo y en primerísimo lugar, que las tres fronteras más violentas de la región son, en el siguiente orden: la de México con Estados Unidos con 74, la de Colombia con Venezuela con 68 y la de Honduras con Guatemala con 65 homicidios". 
TABLA 2

CONOCIMIENTO DE ACCIONES TOMADAS

POR EL ESTADO PARA MINIMIZAR EL CONTRABANDO, SEGÚN ACTITUD FRENTE AL MISMO EN LA ZONA DE FRONTERA

\begin{tabular}{|c|c|c|c|c|c|c|}
\hline \multirow{3}{*}{$\begin{array}{l}\text { Actitud frente al fenómeno del } \\
\text { contrabando }\end{array}$} & \multicolumn{6}{|c|}{ ¿Conoce las acciones tomadas por parte del estado para minimizar el contrabando? } \\
\hline & \multicolumn{2}{|c|}{$\mathrm{Si}$} & \multicolumn{2}{|c|}{ No } & \multicolumn{2}{|c|}{ Total } \\
\hline & $\mathrm{N}$ & $\%$ & $\mathrm{~N}$ & $\%$ & $\mathrm{~N}$ & $\%$ \\
\hline Paciente & 357 & 30.15 & 823 & 69.51 & 1184 & 100,00 \\
\hline Indiferencia & 150 & 20.86 & 568 & 78.99 & 719 & 100,00 \\
\hline Resignación & 248 & 32.54 & 514 & 67.45 & 762 & 100,00 \\
\hline Interés y protesta & 151 & 35.44 & 274 & 64.31 & 426 & 100,00 \\
\hline No comprando productos & 84 & 20.19 & 332 & 79.80 & 416 & 100,00 \\
\hline Otra & 100 & 35.08 & 185 & 64.91 & 285 & 100,00 \\
\hline Total & 1090 & 28.74 & 2696 & 71.09 & 3792 & 100,00 \\
\hline
\end{tabular}

Collins (1995) y Perus (2005) el centro del enfoque consiste en apreciar los conflictos como fruto de un proceso situacional, donde las características de las circunstancias de violencia condicionan el curso de los acontecimientos en la frontera.

Entre las personas que informan que su actitud es no comprando productos son $80 \%$ que declaran que no conocen las acciones estatales (Tabla 2). Estos últimos suman 71\%, o sea, ignoran las acciones gubernamentales para minimizar la economía informal y el contrabando en gran parte sostenida por el transbordo transfronterizo de mercancías. Estamos tentados a suponer que, están tan acostumbrados a tener que socorrer a la nación con medios propios, el pueblo ya se ha desinteresado de las fórmulas y reglas oficiales. Prefiere las suyas. Participan de la disimulación, de atención a detalles inexpresivos de la cultura voraz del consumo, del "juego del contento" a que los explicadores de lo inexplicable recurren, valiéndose de la célebre fórmula de ver signos positivos incluso en las grandes adversidades. En la zona fronteriza del contrabando los ciudadanos están sujetos a una mira extensiva e intensiva y siempre bajo sospecha implícita, en función de la característica de la búsqueda por la seguridad en el capitalismo tardío. En este sentido,

hay una internalización de la frontera ocurriendo con el resurgimiento del capitalismo tardío como estado de seguridad, transformando espacios civiles en fronteras, también la elaboración del sujeto político moderno tardío a través de términos que son únicos y profundamente anclados, pero no intuitivos. Bajo estas condiciones, una biossocialidad altamente internalizada e individualizada se convierte en un sustituto para las convenciones civiles o democráticas de la sociabilidad política (Chalfin, 2012: 296). 
No obstante, los pactos sociales pueden ser favorables para la sociedad cuando no se refieren a la ilegalidad, pues pueden llegar a ser para los entes gubernamentales y las instituciones sociales en general una "nueva modalidad de política pública orientada a promover procesos colectivos y negociados de desarrollo local" (Carmona, 2006: 177), deben ser incorporados en la lucha contra el contrabando de esta zona. La emergencia de pactos sociales y especialmente ilegales ${ }^{11}$ surgen no solo por las diferentes problemáticas que enfrentan sus habitantes, sino que también se sustenta en acciones de ingobernabilidad y problemas del mercado que son los que llevan a sus pobladores a crear acuerdos con los cuales satisfagan en algún modo sus demandas.

\section{Pactos ante el fenómeno social del contrabando}

Se considera la existencia de interacciones socio-espaciales (de naturaleza sociopolítica, por las asimetrías económicas, legales, aspectos institucionales y ético culturales), en las fronteras de América Latina (Carrión, 2011; Caraballo, 2017). Al implicar los pactos sociales, el respeto mutuo entre las personas, los compromisos de quienes pactan y el intercambio entre los actores que hacen vida en zona, es inevitable entender, sí en la zona de frontera concurren pactos sociales para la convivencia, aceptación e intervención en conductas ilegales. En este caso, se trata la existencia de pactos o acuerdos en la región fronteriza relacionados con contrabando, corrupción, soborno y acuerdos entre los actores (Carrión, 2011; Ávila et alii, 2012; Stein et alii, 2015). Por su vez Flores y Pacheco (2017) abordan un tema que nosotros no examinamos que son las migraciones laborales colombo-venezolanas en la frontera Táchira-Norte de Santander.

De un modo general un pacto social de acordó con Sánchez en un país donde el poder político está fluido o diluido,

solo en parte se encuentra en la institucionalidad del Estado, pero difuso en organización de pequeños actores en amplias redes con ingresos propios, servicios de beneficencia, normas de conducta o lealtad, capacidad para hacer alianzas (Sánchez, 2015: 76).

Para Matteucci (1988) si el pacto consiste en relaciones con obligaciones entre las partes, convendría interrogar igualmente cuáles son las sanciones previstas para quienes infringen las reglas del juego. Esto inmediatamente traslada al debate otro problema en una sociedad de relaciones asimétricas: quien detiene el poder o el monopolio de la fuerza y quien renuncia o no al ejercicio de la fuerza, de la violencia.
11. El significado del contrabando (Sánchez, 2015: 77): "en términos prácticos y aplicados, estos debates conceptuales entran en acción y toman vital relevancia en contextos de conflictos sociales, que se presentan, precisamente, por la indiscriminada utilización de estos conceptos, así como por su mala interpretación. Así, entonces, un entendimiento y manejo claro de estos conceptos se tornan relevantes cuando de forma concreta tratamos de organizar los espacios que nos rodean; acciones que involucran delimitación y construcción territorial". 
Dentro de la multiplicidad de acciones sociales podemos ver las interacciones simbólicas y los procesos rituales como herramientas que las personas utilizan para marcar puntos: hacer los contactos apropiados, construir puentes, causar embarazos o incluso destruir a los rivales. Los rituales poseen una analogía, de acuerdo con Collins,

realizamos performances, pero ellas exigen la utilización de un figurín y de un escenario reales: ropa, el escenario, una audiencia y un lugar donde los actores pueden guardar sus equipamientos (Collins, 1995: 114).

En este sentido, se conforman relaciones sociales amparadas en prácticas de violencia múltiple, por veces sin el control del Estado de derecho.

Esta forma de violencia es relativamente nueva en la zona. El 12 de septiembre de 2009 se produjo el asesinato, por sicariato, del alcalde del municipio Panamericano cercano a la frontera de Táchira por parte de paramilitares o "paracos," como se les denomina en la zona; éste último parece indicar el surgimiento de violencia política, característica de la Colombia de los años ochenta y noventa. Este tipo de violencia es significativa del grado de impunidad mientras que los cuerpos de seguridad del Estado, incluyendo la provisión de justicia, han abandonado sus espacios naturales de actuación y le han dejado a los grupos violentos las labores de vigilancia, saneamiento y seguridad (Bustamante, 2011: 216).

En realidad hay mayor concentración (81,46\%) no cruce de muy en desacuerdo cuanto a la confianza en las instituciones del poder público para limitar el contra-

TABLA 3

CONOCIMIENTO DE ACCIONES TOMADAS POR EL ESTADO PARA MINIMIZAR LA ECONOMÍA INFORMAL, SEGÚN CONFIANZA

EN LAS INSTITUCIONES DEL PODER PÚBLICO PARA LIMITAR EL CONTRABANDO

\begin{tabular}{|l|c|c|c|c|c|c|}
\multirow{2}{*}{$\begin{array}{l}\text { Confianza en las instituciones } \\
\text { del poder público para limitar } \\
\text { el contrabando }\end{array}$} & \multicolumn{4}{|c|}{$\begin{array}{c}\text { ¿Conoce las acciones tomadas por parte del Estado } \\
\text { para minimizar la práctica del contrabando? }\end{array}$} \\
\cline { 2 - 7 } & \multicolumn{2}{|c|}{$\mathrm{Si}$} & \multicolumn{3}{|c|}{ No } & \multicolumn{3}{c|}{ Total } \\
\cline { 2 - 7 } & $\mathrm{N}$ & $\%$ & $\mathrm{~N}$ & 72.35 & 123 & 100,00 \\
\hline Muy de acuerdo & 34 & 27.64 & 89 & 70.08 & 468 & 100,00 \\
\hline Ni de acuerdo ni en desacuerdo & 212 & 31.50 & 461 & 68.49 & 673 & 100,00 \\
\hline En desacuerdo & 537 & 33.23 & 1075 & 66.52 & 1616 & 100,00 \\
\hline Muy en desacuerdo & 169 & 18.53 & 743 & 81.46 & 912 & 100,00 \\
\hline Total & 1090 & 28.74 & 2696 & 71.09 & 3792 & 100,00 \\
\hline
\end{tabular}

Fuente: elaboración propia. Datos provenientes de las encuestas aplicadas en Norte de Santander y Táchira. 
bando y a declaración de que no conoce las acciones tomadas por parte del Estado para minimizar la práctica del contrabando (Tabla 3). La mayoría de los habitantes del Norte de Santander sin importar el grupo de edad, están muy de acuerdo y de acuerdo que se visualizan pactos sociales informales en la zona de frontera Norte de Santander y Táchira, es decir existen acuerdos entre personas y/o instituciones relacionados con el tránsito de mercancías de contrabando, las mismas pueden transitar sin el pago de impuestos o aranceles. Taylor (2007) menciona que éstas, en realidad, son fronteras artificiales o que existen singularidades fronterizas.

En el Tabla 4 las actitudes frente al fenómeno de contrabando están en orden decreciente y revelan la apatía ante lo que parece un problema social relevante, una vez que paciencia, indiferencia y resignación se encuentran en la cima de las opciones seleccionadas. Mientras "interés y protesta" y "no comprando productos de contrabando" están en posición secundaria, lo que puede inducir la idea de que hay más concordancia con el contrabando que oposición popular.

La Tabla 4 igualmente indica que, además del desconocimiento de las acciones, rige una baja confianza en las instituciones del poder público para limitar el contrabando. En este sentido, el proceso de globalización parece disolver las fronteras tradicionales de los Estados nacionales, debido a la reforma de las instituciones, a la apertura de los mercados y a la revolución científico-tecnológica en el ámbito de las comunicaciones que se viven a escala planetaria. Sin embargo, no se trata del fin de los límites nacionales sino de la transformación de los mismos, cuestión que hace pensar en la existencia de nuevos rasgos de las fronteras, tal como afirma Marc

TABLA 4

CONFIANZA EN LAS INSTITUCIONES DEL PODER PÚBLICO PARA LIMITAR CONTRABANDO SEGÚN ACTITUD FRENTE AL FENÓMENO DEL CONTRABANDO ENTRE PERSONAS Y/O INSTITUCIONES EN LA ZONA DE FRONTERA

\begin{tabular}{|c|c|c|c|c|c|c|c|c|c|c|c|c|}
\hline \multirow{3}{*}{$\begin{array}{l}\text { Actitud frente al fenómeno del } \\
\text { contra bando }\end{array}$} & \multicolumn{12}{|c|}{ Hay confianza en las instituciones del poder público para limitar el contrabando } \\
\hline & \multicolumn{2}{|c|}{ Muy de acuerdo } & \multicolumn{2}{|c|}{ De acuerdo } & \multicolumn{2}{|c|}{$\begin{array}{l}\text { Ni de acuerdo ni } \\
\text { en desacuerdo }\end{array}$} & \multicolumn{2}{|c|}{ En desacuerdo } & \multicolumn{2}{|c|}{$\begin{array}{c}\text { Muy en } \\
\text { desacuerdo }\end{array}$} & \multicolumn{2}{|c|}{ Total } \\
\hline & $\mathrm{N}$ & $\%$ & $\mathrm{~N}$ & $\%$ & N & $\%$ & $\mathrm{~N}$ & $\%$ & $\mathrm{~N}$ & $\%$ & $\mathrm{~N}$ & $\%$ \\
\hline Paciencia & 40 & 3.37 & 159 & 13.42 & 193 & 16.30 & 527 & 44.51 & 265 & 22.38 & 1184 & 100,00 \\
\hline Indiferencia & 25 & 3.47 & 111 & 15.43 & 177 & 24.61 & 302 & 42.00 & 104 & 14.46 & 719 & 100,00 \\
\hline Resignación & 16 & 2.09 & 98 & 12.86 & 127 & 16.66 & 353 & 46.32 & 168 & 22.04 & 762 & 100,00 \\
\hline Interés y protesta & 21 & 4.92 & 28 & 6.57 & 34 & 7.98 & 180 & 42.25 & 163 & 38.26 & 426 & 100,00 \\
\hline $\begin{array}{l}\text { No comprando productos de } \\
\text { contrabando }\end{array}$ & 12 & 2.88 & 37 & 8.89 & 57 & 13.70 & 158 & 37.98 & 152 & 36.53 & 416 & 100,00 \\
\hline $\mathrm{Ns} / \mathrm{Nr}$ & 9 & 3.15 & 35 & 12.28 & 85 & 29.82 & 96 & 33.68 & 60 & 21.05 & 285 & 100,00 \\
\hline Total & 123 & 3.24 & 468 & 12.34 & 673 & 17.74 & 1616 & 42.61 & 912 & 24.05 & 3792 & 100,00 \\
\hline
\end{tabular}

Fuente: elaboración propia. Datos provenientes de las encuestas aplicadas en Norte de Santander y Táchira. 
12. Por ejemplo, asevera Stein et alii (2015: 56) "en Costa Rica, el $35 \%$ de la población admite haber comprado un producto del contrabando".
Augé (2007: 22): "Las fronteras nunca llegan a borrarse, sino que vuelven a trazarse". Lo que sucede es que la globalización termina por confrontar las heterogeneidades, en todas sus expresiones y, al hacerlo, no diluye las diferencias sino que las traza de manera diferente (Carrión, 2011).

Atestigua Stein et alii (2015: 56) señala "la tolerancia o aceptación hacia el consumo de bienes producto del comercio ilícito ${ }^{12}$ en los países de la región, es un hecho innegable extendido". Los pactos sociales, las reacciones de gobiernos y de actores sociales a la crisis generada por el contrabando, de acuerdo con Avdagic (2011) tienen mucho más que ver con la movilización de recursos y de respuestas delante de la legitimación para mitigar la crisis.

De manera controvertida, es posible explicitar de modo más o menos inequívoco que existe un establecimiento de pacto social conformado por las lógicas circulantes de diferentes actores que luchan con la finalidad de mantenerse en actividad en un escenario adverso. Para Coleman (1990) las personas actúan de forma intencional dirigidas a fines racionales; también eligen acciones o bienes que maximicen sus resultados. Para el caso en examen las acciones pueden llevar a consecuencias y resultados que las personas no buscaban ni preveían; por lo tanto generando efectos contrarios a las intenciones de maximizar sus preferencias. Por las prácticas de contrabando, al atravesar fronteras nacionales, de cierta forma, se hacen partícipes de dos naciones o de ninguna de ellas, razón por la cual la noción de estado-nación se vuelve problemática (Kearney, 1991).

Los pactos son establecidos entre los diferentes actores involucrados como los comerciantes/vendedores, autoridades policiales y militares, contrabandistas y grupos irregulares que controlan las rutas del tránsito de las mercancías, que fomentan el contrabando en la frontera Norte de Santander - Táchira. Estos pactos no pueden controlarse debido a la impunidad que trae el contrabando y su aceptación social. Nosotros no nos concentramos tampoco en la explicación de actitudes diferentes, pero conectadas: la aprobación individual de personas demandando hacer justicia por sus propias manos y la acción policial violando procedimientos legales para capturar sospechosos como si fueran infractores.

Los pactos surgen de acuerdos, se plasman a través de normas morales, sociales o legales. Cuando se trata de pactos sociales se materializan a través de políticas sociales informales (legales o ilegales). En el presente estudio se evidencian pactos sociales en las dinámicas económicas, sociales y culturales que ocurren en el espacio y territorio fronterizo. Las normas que regulan las relaciones sociales están socialmente reconocidas y consolidadas por medio de un pacto social para ejecutar 
estrategias de convivialidad, al mismo tiempo incluido que son utilizadas otras formas de control social que no es sólo lo legal formal.

\section{Consideraciones finales}

De los resultados obtenidos, puede pensarse que las políticas orientadas al crecimiento económico local tienen potencial enfriador sobre el ritmo del conflicto en el campo, lo que, teóricamente, significa un aumento del costo de oportunidad del uso de la violencia (intercambios involuntarios) con respecto al uso del mercado (intercambios voluntarios) para la obtención de ganancias de bienestar. La percepción de pactos sociales informales o acuerdos entre personas y/o instituciones relacionados con el contrabando en la frontera, hacen parte de la dinámica social la cual involucra la población haciendo que se perciban estos pactos. Una de las dinámicas de la frontera son las actividades criminales e ilegales desarrolladas por grupos armados que operan al margen de la ley, que hacen control social en distintos puntos o pasos informales del límite de la frontera colombo venezolana y están inmersos en negocios ilícitos entre ellos el contrabando; actividades que afectan a toda la población, aunque muchos de los contrabandistas que comercializan mercancías en pequeñas cantidades, no formen parte directamente de las mismas, sino simplemente es una fuente de ingresos para vivir debido a los niveles de desempleo que se registran en esta zona fronteriza. Las personas conocen las actividades ilícitas, actores, riesgos y consecuencias y algunos jóvenes ven en estés movimientos un ejemplo a seguir o un espacio de oportunidad en el mercado laboral.

Las relaciones sociales en la frontera están en tensión porque se asocian con formas de cooperación y reciprocidad de un lado y de otro con corrupción, soborno y extorsión. Estos compromisos nacen y se propagan por aspectos institucionales y ético culturales, así como asimetrías jurídicas y económicas y elementos sociopolíticos (Carrión, 2011). Las cuestiones de las fronteras entre países en América Latina son demarcadas y se encuentran en vinculación a una cultura con los desafíos de sobrevivencia y la agenda de derechos.

Las relaciones sociales y los pactos implícitos o explícitos que desarrollan los grupos de ciudadanos hacen que se fomente e institucionalice en la interacción unas relaciones de cooperación y reciprocidad frente a metas pactadas. En este sentido, las mismas motivan las acciones individuales de sus miembros, sin que exista una percepción histórica de ellos, sino que factores extrínsecos como el territorio y la cultura van haciendo que aparezca cierto alineamiento social frente a creencias y percepciones. Por lo anterior, cabe tener en cuenta el espacio con sus conflictos y la territorialidad fronteriza, para estudiar la comprensión de los derechos que sostie- 
nen los pactos, que en torno a la búsqueda de trabajo o ingresos de sobrevivencia, han venido construyendo los habitantes de la frontera del Norte de Santander (Colombia) y de Táchira (Venezuela). Considerando el espacio en la frontera se diseñó la existencia de diferencias en cuanto a la percepción de un ambiente de ilegalidad y violencia, cuya realidad es una de las provocaciones para la existencia de pactos sociales formales o informales en la zona de frontera. Se indagóla relación entre la percepción de los derechos al trabajo, los pactos sociales y las actividades ilegales como parte de la dinámica social, cultural y económica en la frontera referida.

De los ciudadanos en la condición de desempleo o en el subempleo y, como tal, sólo con oportunidades marginales en el mercado, puede comprenderse el rechazo por trabajar como descalificados. El contrabando de mercancías y su comercialización surge como una ventana de oportunidades, sin entrar en el mérito de preferencias. En función de este fenómeno social complejo, emerge en las zonas de frontera lo que podemos calificar como territorios marginales al mundo instituido. La confianza pública de un pacto social sobre los impactos de una especie de pasaporte para la pobreza y sus formas de marginalidad. En consecuencia del cierre de oportunidades, entra en escena la sinergia entre la marginalidad económica, cultural y social.

Puede verse que todos los ciudadanos encuestados de la frontera son sensibles al hecho y los pactos que giran en torno a este. Para el caso de Táchira-dado que es común encontrar productos circulando de manera ilegal en las calles, noticias sobre contrabando y los involucrados en la práctica, vecinos y conocidos que hacen parte activa del ilícito, etc. Esto hace que se cree una relación directa frente a esta percepción, en donde la coyuntura política, el desabastecimiento, las formas de operar de los contrabandistas, las dinámicas sociales y familiares para conseguir los recursos básicos-así sean de contrabando- han hecho que los pactos sean percibidos de manera más clara, sin que incida el nivel educativo en una realidad tan palpable.

Por esto, los resultados hacen pensar las dimensiones políticas en una perspectiva proactiva para engendrar impactos o la comprensión de los derechos en la región. Si hay contrabando que afecta aspectos económicos nacionales, esto no parece motivo para que las demandas locales de la población por efectividad de políticas públicas y las dinámicas transfronterizas, sean sistemáticamente ignoradas en la negociación de los conflictos. A pesar del proceso de globalización, conflictos fronterizos se multiplican y se rediseñan como una instancia por razones singulares o debido a la tensión local/nacional/global. 
En los puntos cruciales de desplazamiento de las personas, las mercancías suben y descienden de valor, a medida que se mueven cruzando la frontera entre Venezuela y Colombia. El valor se altera de acuerdo con una categorización de mercancías como legítimas o ilegítimas y las personas de acuerdo con movimientos legitimados o contrabandistas. Los sistemas de vigilancia revelan tendencias en la gobernanza de los flujos, así como auxilian en el entendimiento de la construcción socio-política de esta valoración y la incesante demarcación de desigualdades en las fronteras en época supuestamente marcada por el libre comercio y globalización.

Por último, a partir de la noción de que las percepciones son derivadas de lo que es socialmente percibido, se desprende que la condición social frente al contrabando engendra distintas visiones. Estas giran en torno a los comicios por la implementación de pactos sociales que se dan en relación al contrabando. El crecimiento del contrabando o las actividades ilegales ha generado un conjunto de impactos, pero que se traducen también en una estructura de oportunidades. Por otra parte, la situación contenciosa y los obstáculos de acceso a bienes y servicios urbanos para amplios sectores de la población vienen derivando en conflictos entre los actores involucrados.

\section{Referencias}

ALVAREZ JR., Robert R. The Mexican-US border: the making of an anthropology of borderlands. Annual Review of Anthropology, v. 24, n. 1, p. 447-470, 1995.

ALBORNOZ, Neida et alii. Análisis del contrabando en el Norte de Santander, desde la percepción de los constructos de la institucionalidad. Cúcuta (CO): Ediciones Universidad Simón Bolívar, 2016.

AUGÉ, Marc. Por una antropología de la movilidad. Barcelona: Gedisa, 2007.

AVDAGIC, Sabina. The conditions for pact. A fuzzy set analysis of the resurgence of Tripartite Concertation. In: AVDAGIC, Sabina et alii (Eds.). Pacts in Europe: emergence, evolution, and institutionalization, p. 17-44. Oxford (UK): Oxford University Press, 2011.

ÁVILA, Ariel F. et alii. La frontera caliente entre Colombia y Venezuela. Bogotá: Corporación Nuevo Arco Iris, 2012.

BOBBIO, Norberto. El futuro de la democracia. México: Fondo de Cultura Económica, 2008. 
_. Liberalism and democracy. New York: Verso, 1990.

BRENNA, Jorge. Espacio y territorio: una mirada sociológica. In: REYES, M.; LÓPEZ, A. (Coords.). Explorando territorios, una visión desde las ciencias sociales. México: Universidad Autónoma Metropolitana (UAM), 2012.

BUSTAMANTE, Ana M. La frontera colombo-venezolana: de la conflictividad limítrofe a la global. In: CARRIÓN, Fernando; ESPÍN, Johanna (Coords.). Relaciones fronterizas: encuentros y conflictos, p. 203-221. Quito: Facultad Latinoamericana de Ciencias Sociales (Flacso), 2011.

CARABALLO, Leonardo J. Estudio socioeconómico de los municipios seleccionados para conformar la zona de integración fronteriza. Área Táchira (Venezuela)-Norte de Santander (Colombia). Aldea Mundo, n. 19, p. 55-62, 2017.

CARMONA, Rodrigo.L os pactos territoriales en Italia. Un análisis de la experiencia reciente y sus implicancias en términos de governance. Revista Eure, n. 96(32), p.117-133, 2006.

CARREÑO, Ángela M. Refugiados colombianos en Venezuela: Quince años en búsqueda de protección. Memorias: Revista de Historia y Arqueología desde el Caribe, n. 24, p. 125-148, 2014.

CARRIÓN, Fernando. En el límite de la vida: la violencia fronteriza. In: CARRIÓN, Fernando; ESPÍN, Johanna (Coords.). Relaciones fronterizas: encuentros y conflictos. Quito: Facultad Latinoamericana de Ciencias Sociales (Flacso), p. 77-117, 2011.

CARRIÓN, Fernando; ESPÍN, Johanna. La geografía del delito en la lógica de frontera. in CARRIÓN, Fernando; ESPÍN, Johanna (Coords.).Relaciones fronterizas: encuentros y conflictos. Quito: Facultad Latinoamericana de Ciencias Sociales (Flacso), p. 9-18, 2011.

CHALFIN, Brenda. Border security as late-capitalist "fix". In: WILSON, Thomas M.; DONNAN, Hastings. A companion to border studies, p. 283-300. Hoboken (NJ): Blackwell Publishing, , 2012.

COLEMAN, James. Foundations of social theory. Cambridge (MA): Harvard University Press, 1990.

COLLINS, Randall.Cuatro tradiciones sociológicas. México: Universidad Autónoma Metropolitana.1995

CUNNINGHAM, Hilary; HEYMAN, Josiah. Introduction: mobilities and enclosures at borders. Identities: Global Studies in Culture and Power, v. 11, n. 3, p.289-302, 2004. 
CUNNINGHAM, Hilary. Permeabilities, ecology and geopolitical boundaries. In: WILSON, Thomas M.; DONNAN, Hastings. A companion to border studies, p. 371-386. Hoboken (NJ): Blackwell Publishing, 2012.

DANE. Estimaciones de población 1985-2005 y proyecciones de población 20052020, 2015. Disponible: <http://www.dane.gov.co/index.php/estadisticas-por-te$\mathrm{ma} /$ demografia-y-poblacion/proyecciones-de-poblacion>.

DAS, Veena; POOLE, Deborah. El estado y sus márgenes: etnografías comparadas. Cuadernos de Antropología Social, n. 27, p. 19-52, 2008.

FLORES, Raquel A.; PACHECO, Mary E. Las migraciones laborales colombo-venezolanas en la frontera Táchira-Norte de Santander. Aldea Mundo, n. 4, p. 51-58, 2017.

GAIGER,Luiz I. Conhecer globalmente: um desafio inadiável dos estudos sobre a economia solidária. Otra Economía, v. 8, n. 14, p. 99-111, 2014.

GARCÍA, Jorge et alii. El Táchira en cifras 2015. El contrabando en las ciudades de frontera desde la perspectiva de la institucionalidad. San Cristóbal (CL): Editorial Litho Arte C.A., 2016.

INSTITUTO NACIONAL DE ESTADÍSTICAS (INE). Censo nacional de población y vivienda. Características de las personas. Estructura poblacional. Santiago (CL), INE, 2011. Disponible: <http://www.redatam.ine.gob.ve/Censo2011/index.html>.

KEARNEY, Michael. Borders and boundaries of State and self at the end of empire. Journal of Historical Sociology, v. 4, n. 1, p. 52-74, 1991.

HEYMAN, Josiah. Ports of entry as nodes in the world system. Identities: Global Studies in Cultureand Power, v. 11, n. 3, p. 303-327, 2004.

MARTINS, J. S. Fronteira: a degradação do outro nos confins do humano. São Paulo: Hucitec, 1997

MATTEUCCI, Nicola. Contratualismo. In: BOBBIO, Norberto; MATTEUCCI, Nicola; PASQUINO, Gianfranco, p. 272-283. Diccionario de política. México: Siglo Veintiuno, 1988.

MURRIETA, Ramón L. M. Espacio social y urbano: visiones de las experiencias y prácticas de sus residentes en ciudad Juárez. In: CARRASCO-GALLEGOS, Brisa V. (Coord.). Megaproyectos urbanos y productivos. Impactos socio-territoriales, p. 129-144. Toluca (MX): Universidad Nacional Autónoma de México (Unam), 2017. 
NAVARRO, María P. B.; PÉREZ, Alfonso L. Territorialidad y políticas públicas. In: REYES, M.; LÓPEZ, A. (Coords.). Explorando territorios, una visión desde las ciencias sociales. México: Universidad Autónoma Metropolitana (UAM), 2012.

OBERG, Perola et alii. Disrupted exchange and declining corporatism: government authority and interest group capability in Scandinavia. Government and Opposition, n. 46, p. 365-391, 2011.

OFFE, Claus. La atribución del status público a los grupos de interés. In: BERGER, Suzanne (Comp.). Organizing interest in Western Europe. Pluralism, corporatism, and the transformation of politics. Cambridge (UK): Cambridge UniversityPress, 1981.

PERUS, Marcos Cueva. Fronteras y representaciones fronterizas: aproximaciones comparativas entre Estados Unidos y América Latina. Estudios Fronterizos, v. 6, n. 11, p. 9-38, 2005.

RIAÑO-GARZÓN, Manuel et alii. Perceived severity of smuggling at the border of Táchira-North of Santander: health psychology approach. Journal of Border Lands Studies, p. 1-18, 2018.

SÁNCHEZ, Ayala, L. De territorios, límites, bordes y fronteras: una conceptualización para abordar conflictos sociales. Revista de Estudios Sociales, n. 53, p. 175-179, 2015.

SANDOVAL, Juan. Una perspectiva situada de la investigación cualitativa en ciencias sociales. Cinta de Moebio, n. 46, p. 37-46, 2013.

STEIN, Eduardo; SCHWARZBAUER, Annette; RAYO, Mariano. Contrabando y defraudación aduanera en Centroamérica. Guatemala: RED/Fundación Konrad Adenauer, 2015.

TAYLOR, Lawrence. El concepto histórico de frontera. In: AGUILERA, Miguel O. (coord). Antropología de las fronteras: alteridad, historia e identidad más allá de la línea, p. 231-261. Tijuana (MX): El Colegio de la Frontera Norte, 2007.

WEBER, Max. Economy and society. Berkeley (CA): University of California Press, 1978. 